\title{
Shiga-Toxin E. coli Hemolytic Uremic Syndrome: Review of Management and Long-term Outcome
}

\author{
V. J. Harkins ${ }^{1,2} \cdot$ D. A. McAllister ${ }^{2} \cdot$ B. C. Reynolds ${ }^{1}$
}

Published online: 17 January 2020

(C) The Author(s) 2020

\begin{abstract}
Purpose of Review We review the pathophysiology of Shiga-Toxin Enteropathogenic-Hemolytic Uremic Syndrome (STECHUS), strategies to ameliorate or prevent evolution of STEC-HUS, management and the improved recognition of long-term adverse outcomes.

Recent Findings Following on from the preclinical evidence of a role for the complement system in STEC-HUS, the use of complement blocking agents has been the major focus of most recent clinical research. Novel therapies to prevent or lessen HUS have yet to enter the clinical arena. The long-term outcomes of STEC-HUS, similarly to other causes of AKI, are not as benign as previously thought.

Summary Optimizing supportive care in STEC-HUS is the only current recommended treatment. The administration of early isotonic fluids may reduce the severity and duration of STEC-HUS. The role of complement blockade in the management of STEC-HUS remains unclear. The long-term sequelae from STEC-HUS are significant and patients with apparent full renal recovery remain at risk.
\end{abstract}

Keywords Haemolytic uraemic syndrome $\cdot$ Prevention $\cdot$ Volume $\cdot$ Saline $\cdot$ Eculizumab

\section{Introduction}

Shiga-toxin Enteropathic Hemolytic Uremic Syndrome (STEC-HUS) presents as a classical thrombotic microangiopathy triad consisting of microangiopathic haemolytic anaemia, thrombocytopenia and renal impairment [1,2]. Severity ranges from mild biochemical abnormalities to persisting end-stage renal disease with a mortality rate of approximately $3 \%$ [2]. STEC-HUS poses a significant clinical risk to paediatric patients as the leading primary renal cause of acute kidney injury (AKI) [3]. Proposed therapies to prevent the development of STEC-HUS have been studied but their efficacy is undetermined.

The risk to children of developing renal failure following STEC-HUS in the short and medium-term is well understood

This article is part of the Topical Collection on Renal

V. J. Harkins

v.harkins.1@ research.gla.ac.uk

1 The Royal Hospital for Children, Glasgow, Scotland

2 University of Glasgow, Glasgow, Scotland
[4]. However, the long-term risks into adulthood are less well known, particularly for patients whose renal function appears to completely recover during the acute episode; it is unclear whether such patients require any form of long-term surveillance.

This review will focus on the prevention and treatment strategies during the initial phase and the long-term outcome and potential consequences into adulthood for paediatric patients following STEC-HUS.

\section{Pathophysiology}

STEC-HUS is a non-immune, thrombotic microangiopathic haemolytic anaemia and shares clinical features of the disease group, thrombotic microangiopathies (TMA). Classically the smallest vessels, the arterioles and capillaries, are primarily involved. The disease process is initiated by Shiga-toxin (stx) on entry into the circulation via endothelial cells. Cell entry occurs via $\mathrm{Gb} 3$ receptors with subsequent disruption of protein synthesis following inclusion into the endoplasmic reticulum [3].

Endothelial cell death results in oedema, creating shear stress within these thickened microvessels; platelet and fibrin 
accumulation further obstructs blood flow [3]. Platelets are removed from the circulation both by endothelial wall deposition and consumption by the reticulo-endothelial system, leading to thrombocytopenia [1]. Frictional turbulent flow through the thickened capillaries causes a mechanical haemolytic anaemia.

The combination of reduced microcirculatory flow and anaemia leads to multi-systemic ischemia. Though this is most apparent in the kidney, any organ may be affected. Neurological involvement is common, occurring in approximately $25 \%$ of patients, with pancreatic, gastrointestinal, ocular, cardiac, and pulmonary involvement all described [5-8]. Diagnosis is predominantly clinical, supported by a plausible source of exposure to $E$. coli (e.g. farm animals) and by identification of $E$. coli; either on stool culture or via positive antibody on serological testing. Rectal swab analysis by polymerase chain reaction may be useful when a stool sample is not available [9]. Histological confirmation is rarely required.

The role of the complement pathway in STEC-HUS was first suggested almost half a century ago, with the identification that some patients had a low C3 at presentation [10]. Further analysis in small patient cohorts demonstrated increased $\mathrm{C} 3$ breakdown products (i.e. C3b, C3c, C3d and CFB) [11]. C3 was also deposited on $30 \%$ of plateletleucocyte complexes in patients with STEC-HUS compared to $12 \%$ of healthy controls [12]. Shiga-toxin activates complement and binds to factor $\mathrm{H}$ (an inhibitor to the alternative pathway) with a reduction in co-factor responsiveness but no impact on functional ability [13]. More recently, activation of $\mathrm{C} 3$ and $\mathrm{C} 3$ a deposition in the glomerular basement membrane has been associated with podocyte dysfunction, with downregulation of nephrin and other functional proteins [14].

The combination of clinical and laboratory findings supporting a role for complement, and the striking impact of complement blockade in the treatment of the related inherited condition, atypical HUS (aHUS), has led to therapeutic efforts targeting complement in the treatment of STEC-HUS [11, 12, 14-17].

\section{Clinical Indicators of E. coli and Risk of Developing STEC-HUS}

The risk of developing STEC-HUS varies internationally and increases with latitude. The highest incidence is in Argentina (12-14 per 100,000 persons [18]. The United Kingdom (UK) has a moderate incidence of 1.2 per 100,000 but this increases to 4.6 per 100,000 in Scotland; one of the highest rates in the developed world [18-20]. Different factors affect $E$. coli transmission including cattle density, commensal carriage rates, E. coli clade type, rural location, and warm weather followed by rain. These factors partially explain the seasonality of STEC-HUS, with the highest incidence occurring between June and September [1, 21]. One disease-burden modelling study estimated that there are around 4000 cases of STECHUS annually worldwide resulting in around 250 deaths, with half of cases occurring in patients under 16 years of age [22] that model likely underestimated the true incidence of STEC infection.

Identifying paediatric patients at initial presentation who have the greatest risk of adverse outcomes may facilitate early preventative measures and (where necessary) prompt transfer to a centre with dialysis provision [23]. It is estimated that progression from STEC infection to STEC-HUS occurs in $2-14 \%$ of sporadic cases and up to $20 \%$ in outbreaks [1, 24-26]. Watery diarrhoea is typical following $24-72 \mathrm{~h}$ incubation with around $90 \%$ progressing to bloody diarrhoea [27]. STEC-HUS typically presents on median day 7 of illness but can occur up to 14 days following infection, often as GI symptoms are improving [2]. Females were at higher risk in some studies [28] but not consistently [25, 29, 30]. Other presenting features associated with a greater risk of progression to STECHUS include a history of vomiting, fever and bloody diarrhoea although statistical significance varies across studies $[25,31]$. Use of anti-motility agents in the initial illness was associated with prolonged bloody diarrhoea but did not reach statistical significance regarding the risk of developing STECHUS [25].

Laboratory findings which are associated with a higher risk of developing STEC-HUS include an elevated white cell count $>13 \times 10^{9} / \mathrm{L}$ and an abnormally high haematocrit (reflecting intravascular depletion) [2, 25, 32].

Clinical risk scores to identify adults with $E$. coli gastroenteritis who are at greater risk for STEC-HUS have been developed, however, the applicability of these tools in children is unknown $[31,33]$.

\section{Treatment}

Supportive therapy remains the mainstay of treatment of STEC-HUS, managing fluid balance, electrolyte abnormalities and hypertension if present [34]. Up to $80 \%$ of patients will require transfusion with blood or platelets during their illness [1]. Oligoanuric AKI, fluid overload, refractory hyperkalaemia or uraemia is supported with renal replacement therapy (RRT), commonly either peritoneal or haemodialysis. A single Cochrane review analysing seven randomized controlled trials (RCTs) showed no benefit of additional therapies such as plasmapheresis over best supportive care [34].

\section{Management with Preventative Volume Expansion}

There is no standardized clinical approach to fluid management in STEC-HUS. In oligoanuric patients, usual management is restriction of intake to measured urinary output and insensible losses. Fluid management of the patient who 
maintains a urine output is influenced by clinical concerns regarding deterioration in renal function and potential fluid overload often resulting in conservative rehydration. However, intravascular depletion may be expected to aggravate any TMA process therefore optimizing hydration could be beneficial.

An initial study in 2004 prospectively tested the hypothesis that the microangiopathic process could be ameliorated by volume expansion leading to improved renal perfusion and thus reduce progression to oligoanuria [24]. This study identified that oligoanuric patients received significantly less sodium and overall volume than non-oliguric patients, with increased statistical significance when analysing the first 4 days of illness. Volume expansion with IV isotonic saline was determined to be potentially nephroprotective, recommending pre-emptive IV fluid administration in children with confirmed $E$. coli infection [24]. It was notably suggested that oral fluids were not an adequate substitution for IV hydration [24].

A multicentre prospective observational cohort study explored this further, confirming the benefits of IV fluid administration in the first 4 days. $84 \%$ of patients who did not receive IV fluids developed oligoanuria, compared to $52 \%$ who did (relative risk(RR) of 1.6 (CI 1.1-2.4)) [35]. The median volume of IV fluid given was $1.7 \mathrm{l} / \mathrm{m}^{2}$, and no patients developed fluid overload. Though that study supported the recommendation for early administration of isotonic saline, no comment was made on duration, rate or volume of fluid.

Two later retrospective observational studies evaluated the relationship between dehydration and outcome. One study found clinically dehydrated patients (defined by World Health Organization classification) were more likely to receive RRT; $71 \%$ in the dehydrated group compared to $41 \%$ of adequately hydrated patients. The number of dialysis days was 50\% higher, (12 versus 8 days), suggesting reduced intravascular volume may exacerbate or accelerate the STEC-HUS disease process [32]. The second study associated high haematocrit at presentation with poorer neurological outcome [36]. As neurological involvement in HUS is strongly associated with mortality, it may be speculated that haemoconcentration and decreased intravascular volume may be similarly associated. The same authors subsequently reported a relationship between IV volume expansion and improved overall outcome on retrospective review, with reduction in need for dialysis, intensive care, development of neurological sequelae and less hospital days [37].

A meta-analysis including the above studies identified benefit of IV fluids up until the day of development of STEC-HUS with an association with reduced need for RRT (Odds Ratio(OR) 0.26 [95\% $0.11-0.6]$ ) and a haematocrit $>23 \%$ was associated with increased risk of oligoanuric STEC-HUS (OR 2.38), RRT (OR 1.9) and death $(\mathrm{OR} 5.13)$ [38••].
The evidence to support IV fluid hydration in the prodromal phase of STEC-HUS continues to grow, yet fluid hydration in at-risk children is not widely practiced. In centres with a high-risk of STEC-HUS, $40 \%$ of patients who developed the syndrome attended paediatric emergency departments early in the disease, however, stool culture and pre-emptive IV fluid administration have not improved over the last 17 years [39॰]. There is a need for increased awareness in early identification of $E$. coli and initiation of IV fluid therapy in primary and secondary care to reduce disease burden globally [39॰].

IV fluid administration in the context of STEC infection does require close monitoring for development of renal impairment and fluid overload and there is an associated cost implication of admission for hydration; however, this should be balanced with the significant morbidity of CKD, ESRD and death. In the absence of curative STEC treatment, comparing different rates of IV fluids would be important to reduce the progression of STEC-HUS, and could be addressed by an RCT.

\section{Antibiotics}

The role of antibiotics in STEC-HUS remains unclear. Worsening of disease is hypothesised to occur following antibiotic administration due to either the widespread release of Shiga-toxin following bacterial cell death, or alteration of the commensal gut flora allows Shiga-toxin to freely attach to the intestinal wall. The contrasting hypothesis is that earlier destruction of $E$. coli leads to reduced excretion of Shiga-toxin and subsequently decreased severity of STEC-HUS. Understanding is complicated further by the differential effects of various antimicrobial agents on different strains of E. coli. Study heterogeneity presents challenging evaluation of results; indeed, meta-analyses of the same combined cohorts can reach opposing conclusions [40, 41, 42•, 43].

Several papers have shown no association between antibiotic use and development of STEC-HUS secondary to E. coli $O 157[25,44,45]$. An RCT in 47 paediatric patients analysed trimethoprim use in E. coli 0157 and showed no significant progression to STEC-HUS, symptomatic improvement or change in Shiga-toxin excretion [44]. Although antibiotic use in hospital did not reach statistical significance as a risk factor for STEC-HUS, pre-hospital administration of antibiotics was associated with increased risk [45].

Increased risk was also identified in a 15-year study of STEC-HUS incorporating 783 patients aged $<18$ years, in whom recent respiratory tract infection and treatment with antibiotic was associated with an increased mortality [2]. In a prospective study of 71 paediatric patients with $E$. coli 0157:H7, antibiotic use was a risk factor for progression to STEC-HUS [46]. Bactericidal antibiotics, (in particular Blactams) administered in the first 3 days of illness was associated with increased risk of STEC-HUS in a case-control study 
of 195 patients (OR 12.4 and 11.3) [47]. One study comparing the response of different $E$. coli subtypes to antibiotic therapy (namely, ciprofloxacin, meropenem, fosfomycin or chloramphenicol) showed release of Shiga toxin following antibiotics by E. coli O157:H7 but not subtype O104:H4 [48].

An in vivo mouse model using E. coli 086 demonstrated decreased Shiga-toxin production and reduced mortality following administration of azithromycin [49]. Following the large German E. coli 0104:H4 outbreak, a retrospective analysis identified that use of azithromycin reduced duration of Shiga-toxin excretion in stool [50]. Other retrospective analysis of the same outbreak identified patients treated with dual antibiotic therapy (specifically ciprofloxacin and meropenem IV) significantly shortened the duration of excretion of Shiga-toxin in stool, with a concomitant lower incidence of seizures and death compared to patients who did not receive antibiotics [51]. Confounding factors included the absence of criteria for commencing antibiotics or whether the severity of disease influenced antibiotic administration [51]. A further sub-analysis of the outbreak found significant reduction in STEC-HUS development with ciprofloxacin though this was under-powered to definitively reach a conclusion [52]. A multicenter observational study of fosfomycin use in STEC infection identified a possible reduction to progression to STEC-HUS when given within the first 5 days but would benefit from supporting studies(OR 0.15 [95\%CI 0.05-0.45]) [53].

One meta-analysis did not find an association between antibiotic use and STEC-HUS [43]. Exclusion of studies at high risk of bias in a more recent meta-analysis conversely concluded that antibiotic use was associated with increased risk of STEC-HUS [42•]. In contrast, a systematic review identified the potential benefit of antibiotics, namely azithromycin, in inhibiting cell wall and protein synthesis and recommended use in specific circumstances [41].

A consensus on antibiotics has not been established. It is likely that differential effects are seen between microbial strains and choice of antibiotic. Use of antimicrobials should be considered on an individual and strain basis only when benefit is thought likely. Future characterization of the strain response, in particular during outbreaks may permit more informed decisions [48].

\section{Platelet Transfusion}

Platelet transfusion has historically been relatively contraindicated in STEC-HUS due to the theoretical potential to aggravate the thrombotic microangiopathy, administration being restricted to transfusion immediately preceding surgical interventions and in response to significant haemorrhage or mucosal bleeding. Two retrospective case-control studies [54, 55], demonstrated no differences comparing patients who did or did not receive platelets in disease severity, neurological complications, requirement for intensive care, or mortality. A small rise in inflammatory markers was noted following platelet transfusion in the second study; these returned to baseline [55]. It was also noted that 6 patients died during the German E. coli 0104:H4 outbreak from procedure-related bleeding [55]. Though larger studies are required to fully reassure, the risks of platelet transfusion may not be as high as previously theorized, and may confer clinical benefit, especially in patients at greater risk of haemorrhage [55].

\section{Eculizumab}

Eculizumab is a monoclonal antibody that targets complement $\mathrm{C} 5 \mathrm{~b}$ and is a licenced effective treatment for aHUS, which shares many disease features with STEC-HUS. This prompted further investigation into a possible role in the management of STEC-HUS.

In a case series of three patients in 2011, severe neurological involvement led to compassionate treatment with eculizumab. There was resolution of neurological findings within $24 \mathrm{~h}$ and improvement of platelets and lactate dehydrogenase (LDH) within 5 days [56]. This led to increased 'compassionate' use in some centres, especially where neurological involvement was considered severe, with an escalation of usage during the outbreak of E. coli O104:H4 in 2011.

That outbreak dominates the current literature surrounding the use of eculizumab in the treatment of STEC-HUS. The differential ability of centres to provide eculizumab alongside plasmapheresis led to a natural nested cohort study, admittedly with many confounding factors [57]. Unadjusted analyses suggested a higher mortality in the best supportive care cohort, but there was an evident selection bias with a predominance of elderly patients. Treatment allocations were by clinician choice and not randomized or age-matched. When considering severity matched cohorts, there was no evidence for additional benefit from plasmapheresis and eculizumab over supportive care [57].

A further retrospective analysis of this cohort of 298 patients reviewed 67 patients who received eculizumab [51] (Table 1). This cohort presented with more severe disease compared to those in other treatment arms. When matched for severity using patients receiving plasmapheresis, there was no difference in complication rates or recovery time of biochemical markers of HUS. There was no best supportive care model available for comparison [51].

Smaller cohorts from the same outbreak have also been reported from France. A nine patient case series, all receiving plasmapheresis, reported eculizumab administration with neurological and biochemical improvement in all patients [8]. Azithromycin prophylaxis was administered with eculizumab, which as described may also lead to earlier reduction of faecal Shiga-toxin in E. coli 0104:H4 [51]. 


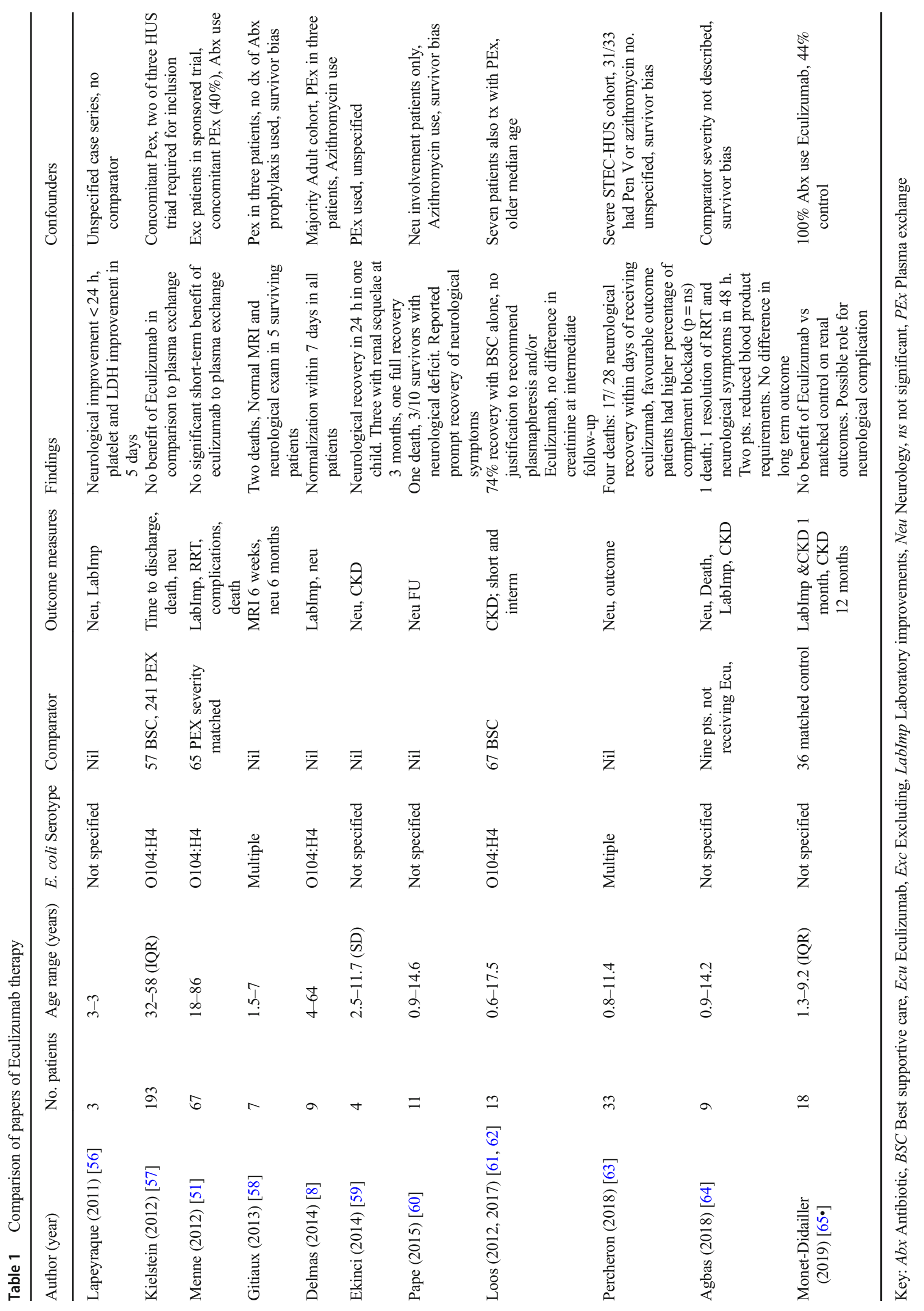


A small study of seven patients receiving eculizumab for treatment of STEC-HUS described the reversibility of Magnetic Resonance Imaging (MRI) cerebral abnormalities in the initial phase of disease when revisited at 6 months [58]. This was a small uncontrolled observational study, MRI scan in the acute phase may be normal despite severe disease and the natural course of transient neurological findings is resolution [66-68], so evidence of definitive benefit is lacking.

Another analysis of 33 French children in the E. coli O104 outbreak (which included seven patients from the above cohort) separated patients retrospectively into groups with a favourable or unfavourable outcome [63]. Analysis of complement blockade at follow-up showed a higher degree of complement blockade in the favourable outcome group (11/ 14 [79\%] versus 9/15 [53\%]). The difference between the two groups did not reach significance. Several limitations in that report (e.g. unclear exclusion criteria) prevent any firm conclusion of clinical benefit $[67,69]$. There is no evidence to date that persistence of complement blockade at 6 months relates to the efficacy of eculizumab in an acute phase of illness [63].

A retrospective case series from Istanbul reported nine paediatric patients with STEC-HUS treated with eculizumab at the discretion of the clinician at a median of 12 days (149 days) - in some cases, after the requirement for RRT had resolved [64]. Baseline characteristics between groups were similar but no descriptor for dialysis requirement or maximal creatinine was reported. Two patients treated with eculizumab were reported to have a reduction in blood product requirements and one child had resolution of neurological involvement and dialysis requirements within $48 \mathrm{~h}$. One child died at day 50 from Gram-negative sepsis following five eculizumab doses [64]. The wide variation and timing of eculizumab administration, loss to follow-up and unclear severity comparators limit the applicability of these data [64].

A recent paediatric retrospective matched control study from France described 18 patients receiving Eculizumab in a single centre. No benefit of Eculizumab was found on renal outcome, however possible benefit was identified in the treatment of neurological STEC-HUS [65•].

Multiple narrative reviews attempting to establish the efficacy of eculizumab in the treatment of STEC-HUS have not established benefit. The need for large RCTs has been recognized, with two such trials now underway $[16,70]$. The UKbased ECUSTEC trial is a double-blind RCT recruiting patients with STEC-HUS to receive eculizumab or saline-based placebo [71••]. The primary aim of the trial is to assess whether early administration of eculizumab will reduce a composite score for severity of STEC-HUS and secondary aims will assess the presence of CKD, neurological sequelae and health-related quality of life at 1 year [71••]. ECULISHU in France is a single-blind RCT to eculizumab or dextrose-based placebo, with patients who deteriorate on placebo converted to the eculizumab arm of the trial [72]. The primary outcome is the duration of acute dialysis. Secondary aims include development of CKD, haematological abnormalities, duration of complement blockade and effect on terminal complement complex (TCC) up to 1 year post-STEC-HUS. The results of these trials will better inform whether eculizumab has a role in the treatment of STEC-HUS.

\section{Long-term Outcome}

Previously, a return to normal renal function after an acute episode of STEC-HUS was felt to have no ongoing sequelae. More recent longitudinal data on patient cohorts with previous AKI of any cause demonstrates that the risks of further renal dysfunction persist over time. [73].

One meta-analysis reviewing the development of longterm renal sequelae after STEC-HUS (with a minimum of 12 months from acute disease onset) reported mortality or end-stage renal disease (ESRD) in $0-30 \%$ of patients. Up to $64 \%$ of patients developed abnormal GFR, proteinuria or hypertension [4]. Included studies had notable heterogeneity, including importantly the duration of follow-up, with fewer studies persisting into adulthood.

A small cohort of 29 patients examined long-term followup into adulthood (15-25 years). Ten patients had no renal sequelae; the remaining 19 developed renal dysfunction (isolated hypertension $n=7$, proteinuria $n=4$ ) with four reaching ESRD [74]. That cohort also reported that an acute requirement for RRT did not predict either subsequent CKD or those at long-term risk [74]. That finding has been replicated in other studies; deterioration in GFR occurring in patients not requiring dialysis during the acute illness $[75,76]$.

One study reported only those patients who did not require RRT ( $n=130$, mean follow-up 12.2 years), demonstrating a lower rate of sequelae, with $15 \%$ of patients developing hypertension or proteinuria, and $6 \%$ developing an abnormal GFR but no patients reaching ESRD [77].

A medium-term longitudinal study of an initial cohort of 114 patients described progression of CKD in relation to GFR at 1 year. 66 of 92 patients (72\%) had a normal GFR at 1 year. Follow-up data were available for 40 patients at 5 years. Six patients with a previously abnormal GFR had normalized, whilst three additional patients had developed abnormal GFR in the interim period [76]. Using data from the 2011 European $\mathrm{O} 104$ outbreak available for 72 patients at a mean follow-up of 3 years, GFR improved in two of four patients with a previous abnormal GFR; one GFR normalized and a further two patients improved from CKD four to three [62]. These studies demonstrate that patients with less severe initial disease may still be at risk of long-term renal sequelae, consistent with other AKI literature. 
Sub-clinical STEC-HUS may occur in patients not undergoing renal evaluation during the acute STEC infection. Following the E. coli O157 Walkerton outbreak in Ontario, epidemiological studies ( $n=1977)$ identified an increased risk for hypertension, abnormal GFR and self-reported cardiovascular disease when compared to unexposed matched case controls (HR 1.33, 1.15 and 2.13) [78]. These findings were not replicable in a smaller 19 patient paediatric cohort when compared to matched controls 4 years post initial illness [79]. STEC-HUS occurs more rarely in adult populations, and the smaller paediatric sample size, or greater plasticity of paediatric patients may contribute to the disease differences.

There are data supporting the supposition that children with a 'full' renal recovery have ongoing detectable manifestations of renal disease. Two small papers assessed the capacity for hyperfiltration through protein loading and identified a blunting in this response - those authors proposed that this mechanism may contribute to ongoing vulnerability to $\mathrm{CKD}$ despite normalization of renal function, with patients behaving similarly to those with a solitary kidney $[80,81]$. Abnormal endothelial function of skin microvasculature using Doppler fluximetry was found in $50 \%$ of patients who appeared to have fully recovered renal function following STEC-HUS and were normotensive [82]. Clarifying the underlying pathogenesis for these persisting abnormalities may assist in informing future therapies.

\section{Potential Future Therapies}

The mainstay of care remains supportive during an acute episode of STEC-HUS and is predominantly reactive once the disease process is recognized. Both the trials of eculizumab (detailed extensively previously) are also reactive, though with the intention of intervention in the earlier stages of the illness. An alternative target is the immediate response to the toxin itself.

An RCT of an oral Shiga-toxin binding agent showed no benefit, potentially indicating the toxin may have caused established damage during the time delay between symptom onset and identification of STEC infection [83]. The development of monoclonal antibodies against Shiga-toxin 2 (Stx2) has shown some promise in animal models. A piglet model showed neurological benefit of urtoxazumab when administered $24 \mathrm{~h}$ following STEC administration [84], with phase I trials confirming safety of urtoxazumab in a relevant patient population [85]. As yet, clinical efficacy has not been convincingly demonstrated. Research efforts to develop agents for neutralization of Shiga-toxin are ongoing, such as the development of humanized recombinant antibody fragments directed against Stx2 which effectively neutralize cytotoxicity in vitro [86].

Rather than a reactive response, proactively preventing infection through vaccination has been considered [87]. Early approaches to targeting Shiga-toxin $2 \mathrm{~b}$ (Stx2b) were promising but proved difficult to translate to humoral immunity [88-90]. This led to the development of novel genetic approaches, using bacterially derived DNA [91]. This approach uses regulatory gene elements which cause antigenic responses to Stx2B. Though apparently effective in mouse vaccination models, further research was limited by technical issues with production of recombinant Stx2B including inherent instability [92]. Mucosal immunity is an alternative target for therapies as it plays a key role in the pathogenicity of stx. A chimeric protein OmpA-LTB with the ability to bind against the intestinal wall when taken orally allows an immunogenic response to E. coli 0157:H7 to be mounted in silico [93•]. Targeting outer membrane vesicles (OMVs) produced by E. coli O157:H7 has been successfully explored using eyedrop administration in mice [94]. More recently, approaches targeting OMVs have also been tested in cattle and proved effective, providing encouraging results for future human use [95].

\section{Conclusions}

Best supportive care remains the mainstay of treatment of STEC-HUS in paediatric patients. Volume expansion with isotonic saline may be considered in patients with probable STEC, though use is most effective in the first 4 days of symptoms. The role of antibiotics in STEC-HUS remains unclear - trials incorporating analysis by strain may be necessary. Complement blockade is increasingly used, though robust supportive evidence is absent; two ongoing trials may clarify this.

The long-term outcome of STEC-HUS is less optimistic than earlier reports suggested, as longitudinal follow-up reveals patients remain at risk into adulthood. Recognition may allow earlier management of proteinuria and hypertension.

At present, prevention of STEC infection remains the best strategy for reducing complications of STEC-HUS.

\section{Compliance with Ethical Standards}

Human and Animal Rights and Informed Consent This article does not contain any studies with human or animal subjects performed by any of the authors.

Abbreviations $A K I$, acute kidney injury; $a H U S$, atypical haemolytic uraemic syndrome; $C I$, confidence interval; $C K D$, chronic kidney disease; E. coli, Eschieria coli; ESRD, end-stage renal disease; GFR, glomerular filtration rate; $H R$, hazard ratio; $H U S$, haemolytic uraemic syndrome; $I V$, intravenous; $L D H$, lactate dehydrogenase; $M R I$, magnetic resonance imaging; $O M V$, outer membrane vesicles; $O R$, odds ratio; $P C R$, protein creatinine ratio; $R C T$, randomized controlled trial; $R R$, relative risk; $R R T$, renal replacement therapy; STEC, Shiga toxin-producing E. coli; STEC-HUS, haemolytic uraemic syndrome secondary to E. coli 
$O 157$ and other serotypes; Stx, Shiga-toxin; Stx2b, Shiga-toxin 2b; TCC, terminal complement complex; $T M A$, thrombotic microangiopathy; $U K$, United Kingdom

Open Access This article is licensed under a Creative Commons Attribution 4.0 International License, which permits use, sharing, adaptation, distribution and reproduction in any medium or format, as long as you give appropriate credit to the original author(s) and the source, provide a link to the Creative Commons licence, and indicate if changes were made. The images or other third party material in this article are included in the article's Creative Commons licence, unless indicated otherwise in a credit line to the material. If material is not included in the article's Creative Commons licence and your intended use is not permitted by statutory regulation or exceeds the permitted use, you will need to obtain permission directly from the copyright holder. To view a copy of this licence, visit http://creativecommons.org/licenses/by/4.0/.

\section{References}

Papers of particular interest, published recently, have been highlighted as:

- Of importance

•. Of major importance

1. Mele C, Remuzzi G, Noris M. Hemolytic uremic syndrome: HUS. Semin Immunopathol. 2014;36:399-420.

2. Mody RK, Gu W, Griffin PM, Jones TF, Rounds J, Shiferaw B, et al. Postdiarrheal hemolytic uremic syndrome in United States children: clinical Spectrum and predictors of in-hospital death. J Pediatr. 2015;166:1022-9.

3. Keir LS, Marks SD, Kim JJ. Shigatoxin-associated hemolytic uremic syndrome: current molecular mechanisms and future therapies. Drug Des Devel Ther. 2012;6:195-208.

4. Garg AX, Suri RS, Barrowman N, Rehman F, Matsell D, RosasArellano MP, et al. Long-term renal prognosis of diarrheaassociated hemolytic uremic syndrome. JAMA. 2003;290:1360.

5. Gerber A, Karch H, Allerberger F, Verweyen HM, Zimmerhackl LB (2002) Clinical course and the role of Shiga toxin-producing Escherichia coli infection in the hemolytic-uremic syndrome in pediatric patients, 1997-2000, in Germany and Austria: a prospective study.

6. Braune SA, Wichmann D, von Heinz MC, Nierhaus A, Becker H, Meyer TN, et al. Clinical features of critically ill patients with Shiga toxin-induced hemolytic uremic syndrome. Crit Care Med. 2013:41:1702-10.

7. Rigamonti D, Simonetti GD. Direct cardiac involvement in childhood hemolytic-uremic syndrome: case report and review of the literature. Eur J Pediatr. 2016;175:1927-31.

8. Delmas Y, Vendrely B, Clouzeau B, et al. Outbreak of Escherichia coli O104:H4 haemolytic uraemic syndrome in France: outcome with eculizumab. Nephrol Dial Transplant. 2014;29:565-72.

9. Freedman SB, Xie J, Nettel-Aguirre A, Lee B, Chui L, Pang XL, et al. Enteropathogen detection in children with diarrhoea, or vomiting, or both, comparing rectal flocked swabs with stool specimens: an outpatient cohort study. Lancet Gastroenterol Hepatol. 2017;2:662-9.

10. Monnens L, Hendrickx G, van Wieringen P, van Munster P. Letter: serum-complement levels in haemolytic-uraemic syndrome. Lancet (London, England). 1974;2:294.

11. Monnens L, Molenaar J, Lambert P, Proesmans W, van PM. The complement system in hemolytic-uremic syndrome in childhood. Clin Nephrol. 1980;13:168-71.
12. Ståhl A-L, Sartz L, Karpman D. Complement activation on plateletleukocyte complexes and microparticles in enterohemorrhagic Escherichia coli-induced hemolytic uremic syndrome. Blood. 2011;117:5503-13

13. Orth D, Khan AB, Naim A, et al. Shiga toxin activates complement and binds factor $\mathrm{H}$ : evidence for an active role of complement in hemolytic uremic syndrome. J Immunol. 2009;182:6394-400.

14. Zoja C, Buelli S, Morigi M. Shiga toxin triggers endothelial and podocyte injury: the role of complement activation. Paed Nephrol. 2019;34:379-88.

15. Thurman JM, Marians R, Emlen W, Wood S, Smith C, Akana H, et al. Alternative pathway of complement in children with diarrheaassociated hemolytic uremic syndrome. Clin J Am Soc Nephrol. 2009:4:1920-4.

16. Walsh PR, Johnson S. Eculizumab in the treatment of Shiga toxin haemolytic uraemic syndrome. Paed Nephrol. 2018;34:1485-92. https://doi.org/10.1007/s00467-018-4025-0.

17. Buelli S, Zoja C, Remuzzi G, Morigi M. Complement activation contributes to the pathophysiology of Shiga toxin-associated hemolytic uremic syndrome. Microorganisms. 2019. https://doi.org/10. 3390/microorganisms 7010015 .

18. Rivas M, Chinen I, Miliwebsky E, Masana M. Risk factors for Shiga toxin-producing Escherichia coli-associated human diseases. Micro Spect. 2014. https://doi.org/10.1128/microbiolspec.EHEC0002-2013.

19. Sharp JCM, Coia JE, Curnow J, Reilly WJ. Escherichia coli O157 infections in Scotland. J Med Microbiol. 1994;40:3-9.

20. Smith-Palmer A, Hawkins G, Couper S, Maxwell H, Reynolds B, Harkins V, Allison L, Hanson M. Global spread of stec and managing the consequences. In: Int. Netw. Paediatr. Surveill. Units. BMJ Publishing Group Ltd and Royal College of Paediatrics and Child Health; 2018. p. A196.3-A197.

21. Money P, Kelly AF, Gould SWJ, Denholm-Price J, Threlfall EJ, Fielder MD. Cattle, weather and water: mapping Escherichia coli O157:H7 infections in humans in England and Scotland. Environ Microbiol. 2010;12:2633-44.

22. Majowicz SE, Scallan E, Jones-Bitton A, Sargeant JM, Stapleton J, Angulo FJ, et al. Global incidence of human Shiga toxin-producing Escherichia coli infections and deaths: a systematic review and knowledge synthesis HHS public access. Foodborne Pathog Dis. 2014;11:447-55.

23. Oakes RS, Siegler RL, McReynolds MA, Pysher T, Pavia AT. Predictors of fatality in postdiarrheal hemolytic uremic syndrome. Pediatrics. 2006;117:1656-62.

24. Ake JA, Jelacic S, Ciol MA, Watkins SL, Murray KF, Christie DL, et al. Relative Nephroprotection during Escherichia coli O157:H7 infections: association with intravenous volume expansion. Pediatrics. 2005;115:e673-80.

25. Bell BP, Griffin PM, Lozano P, Christie DL, Kobayashi JM, Tarr PI. Predictors of hemolytic uremic syndrome in children during a large outbreak of Escherichia coli O157:H7 infections. Pediatrics. 1997;100:E12.

26. Locking ME, Pollock KGJ, Allison LJ, Rae L, Hanson MF, Cowden JM. Escherichia coli O157 infection and secondary spread, Scotland, 1999-2008. Emerg Infect Dis. 2011;17:524-7.

27. Holtz LR, Neill MA, Tarr PI. Acute bloody diarrhea: a medical emergency for patients of all ages. YGAST. 2009;136:1887-98.

28. Rowe PC, Walop W, Lior H, Mackenzie AM. Haemolytic anaemia after childhood Escherichia coli $\mathrm{O} 157$.H7 infection: are females at increased risk? Epidemiol Infect. 1991;106:523-30.

29. Cimolai N, Basalyga S, Mah DG, Morrison BJ, Carter JE. A continuing assessment of risk factors for the development of Escherichia coli O157:H7-associated hemolytic uremic syndrome. Clin Nephrol. 1994;42:85-9. 
30. Kemper MJ. Outbreak of hemolytic uremic syndrome caused by E. coli O104:H4 in Germany: a pediatric perspective. Pediatr Nephrol. 2012;27:161-4.

31. Hamilton D, Cullinan J. A practical composite risk score for the development of Haemolytic Uraemic syndrome from Shiga toxinproducing Escherichia coli. Eur J Pub Health. 2019. https://doi.org/ 10.1093/eurpub/ckz132.

32. Balestracci A, Martin SM, Toledo I, Alvarado C, Wainsztein RE. Dehydration at admission increased the need for dialysis in hemolytic uremic syndrome children. Pediatr Nephrol. 2012;27:140710 .

33. Zoufaly A, Cramer JP, Vettorazzi E, Sayk F, Bremer JP. Risk factors for development of hemolytic uremic syndrome in a cohort of adult patients with STEC 0104:H4 infection. PLoS One. 2013;8:59209.

34. Michael M, Elliott EJ, Craig JC, Ridley G, Hodson EM. Interventions for hemolytic uremic syndrome and thrombotic thrombocytopenic purpura: a systematic review of randomized controlled trials. Am J Kidney Dis. 2009;53:259-72.

35. Hickey CA, Beattie TJ, Cowieson J, et al. Early volume expansion during diarrhea and relative Nephroprotection during subsequent hemolytic uremic syndrome. Arch Pediatr Adolesc Med. 2011;165:884

36. Ardissino G, Daccò V, Testa S, Civitillo CF, Tel F, Possenti I, et al. Hemoconcentration: a major risk factor for neurological involvement in hemolytic uremic syndrome. Pediatr Nephrol. 2015;30: 345-52.

37. Ardissino G, Tel F, Possenti I, et al. Early volume expansion and outcomes of hemolytic uremic syndrome. Pediatrics. 2016;137: e20152153.

38.• Grisaru S, Xie J, Samuel S, Hartling L, Tarr PI, Schnadower D, et al. Associations between hydration status, intravenous fluid administration, and outcomes of patients infected with Shiga toxinproducing Escherichia coli: a systematic review and meta-analysis. JAMA Pediatr. 2017;171:68 A Meta-analysis of all available papers on the effect of IV hydration.

39. Balestracci A, Meni Battaglia L, Toledo I, Martin SM, Alvarado C. Prodromal phase of hemolytic uremic syndrome related to Shiga toxin-producing Escherichia coli. Pediatr Emerg. 2019; Care 1 analysis of early identification of E.coli and early IV fluid administration; no improvement over the last 17 years.

40. Panos G, Betsi G, Falagas M. Systematic review: are antibiotics detrimental or beneficial for the treatment of patients with Escherichia coli O157:H7 infection? Aliment Pharmacol Ther. 2006;24:731-42.

41. Agger M, Scheutz F, Villumsen S, Re Mølbak K, Munk Petersen A. Antibiotic treatment of verocytotoxin-producing Escherichia coli (VTEC) infection: a systematic review and a proposal. J Antimicrob Chemother. 2015;70:2440-6.

42. Freedman SB, Xie J, Neufeld MS, et al. Shiga toxin-producing Escherichia coli infection, antibiotics, and risk of developing hemolytic uremic syndrome: a meta-analysis. Clin Infect Dis. 2016;62:1251-8 Meta-analysis of antibiotic use excluding papers at high-risk of bias.

43. Safdar N, Said A, Gangnon RE, Maki DG. Risk of hemolytic uremic syndrome after antibiotic treatment of Escherichia coli O157: H7 enteritis a meta-analysis. JAMA. 2002;288:996-1001.

44. Proulx F, Turgeon JP, Delage G, Lafleur L, Chicoine L. Randomized, controlled trial of antibiotic therapy for Escherichia coli O157:H7 enteritis. J Pediatr. 1992;121:299-303.

45. Dundas S, Todd WTA, Stewart AI, Murdoch PS, Chaudhuri AKR, Hutchinson SJ. The Central Scotland Escherichia coli O157:H7 outbreak: risk factors for the hemolytic uremic syndrome and death among hospitalized patients. Clin Infect Dis. 2001;33:923-31.

46. Wong CS, Jelacic S, Habeeb RL, Watkins SL, Tarr PI. The risk of the hemolytic-uremic syndrome after antibiotic treatment of
Escherichia coli O157:H7 infections. N Engl J Med. 2000;342: 1930-6.

47. Smith KE, Wilker PR, Reiter PL, Hedican EB, Bender JB, Hedberg CW. Antibiotic treatment of Escherichia coli O157 infection and the risk of hemolytic uremic syndrome, Minnesota. Pediatr Infect Dis J. 2012;31:37-41.

48. Corogeanu D, Willmes R, Wolke M, Plum G, Utermöhlen O, Krönke M. Therapeutic concentrations of antibiotics inhibit Shiga toxin release from enterohemorrhagic E. coli $\mathrm{O} 104: \mathrm{H} 4$ from the 2011 German outbreak. BMC Microbiol. 2012;12:160.

49. Ohara T, Kojio S, Taneike I, Nakagawa S, Gondaira F, Tamura Y, et al. Effects of azithromycin on Shiga toxin production by Escherichia coli and subsequent host inflammatory response. Antimicrob Agents Chemother. 2002;46:3478-83.

50. Nitschke M, Sayk F, Härtel C, et al. Association between azithromycin therapy and duration of bacterial shedding among patients with Shiga toxin-producing Enteroaggregative Escherichia coli O104:H4. JAMA. 2012;307:1046.

51. Menne J, Nitschke M, Stingele R, et al. Validation of treatment strategies for enterohaemorrhagic Escherichia coli O104:H4 induced haemolytic uraemic syndrome: case-control study. BMJ. 2012;345:e4565.

52. Geerdes-Fenge HF, Löbermann M, Nürnberg M, Fritzsche C, Koball S, Henschel J, et al. Ciprofloxacin reduces the risk of hemolytic uremic syndrome in patients with Escherichia coli O104: H4-associated diarrhea. Infection. 2013;41:669-73.

53. Tajiri H, Nishi J, Ushijima K, Shimizu T, Ishige T, Shimizu M, et al. A role for fosfomycin treatment in children for prevention of haemolytic-uraemic syndrome accompanying Shiga toxinproducing Escherichia coli infection. Int J Antimicrob Agents. 2015;46:586-9.

54. Balestracci A, Martin SM, Toledo I, Alvarado C, Wainsztein RE. Impact of platelet transfusions in children with post-diarrheal hemolytic uremic syndrome. Paediatr Nephrol. 2013;28:919-25.

55. Beneke J, Sartison A, Kielstein JT, Haller H, Nitschke M, Kunzendorf U, et al. Clinical and laboratory consequences of platelet transfusion in Shiga toxin-mediated hemolytic uremic syndrome. Transfus Med Rev. 2017;31:51-5.

56. Lapeyraque A-L, Malina M, Fremeaux-Bacchi V, Boppel T, Kirschfink M, Oualha M, et al. Eculizumab in severe Shiga-toxin-associated HUS. N Engl J Med. 2011;364:2561-3.

57. Kielstein JT, Beutel G, Fleig S, et al. Best supportive care and therapeutic plasma exchange with or without eculizumab in Shiga-toxin-producing E. coli O104:H4 induced haemolyticuraemic syndrome: an analysis of the German STEC-HUS registry. Nephrol Dial Transplant. 2012;27:3807-15.

58. Gitiaux C, Krug P, Grevent D, Kossorotoff M, Poncet S, Eisermann $\mathrm{M}$, et al. Brain magnetic resonance imaging pattern and outcome in children with haemolytic-uraemic syndrome and neurological impairment treated with eculizumab. Dev Med Child Neurol. 2013;55: 758-65.

59. Ekinci Z, Candan C, Alpay H, Canpolat N, Akyüz SG, Gündüz Z, et al. Hemolytic uremic syndrome outbreak in Turkey in 2011. Turk J Pediatr. 2013;55:246-52.

60. Pape L, Hartmann H, Bange FC, Suerbaum S, Bueltmann E, Ahlenstiel-Grunow T. Eculizumab in typical hemolytic uremic syndrome (HUS) with neurological involvement. Medicine (Baltimore). 2015;94:1-6.

61. Loos S, Ahlenstiel T, Kranz B, Staude H, Pape L, Härtel C, et al. An outbreak of Shiga toxin-producing Escherichia coli O104:H4 hemolytic uremic syndrome in Germany: presentation and short-term outcome in children. Clin Infect Dis. 2012;55:753-9.

62. Loos S, Aulbert W, Hoppe B, Ahlenstiel-Grunow T, Kranz B, Wahl $\mathrm{C}$, et al. Intermediate follow-up of pediatric patients with hemolytic uremic syndrome during the 2011 outbreak caused by E. coli O104: H4. Clin Infect Dis. 2017;64:1637-43. 
63. Percheron L, Gramada R, Tellier S, et al. Eculizumab treatment in severe pediatric STEC-HUS: a multicenter retrospective study. Pediatr Nephrol. 2018;33:1385-94.

64. Ağbaş A, Göknar N, Akıncı N, Yıldırım ZY, Taşdemir M, Benzer $\mathrm{M}$, et al. Outbreak of Shiga toxin-producing Escherichia-coliassociated hemolytic uremic syndrome in Istanbul in 2015: outcome and experience with eculizumab. Pediatr Nephrol. 2018;33: 2371-81.

65. Monet-Didailler C, Chevallier A, Godron-Dubrasquet A, Allard L, Delmas Y, Contin-Bordes C, et al. Outcome of children with Shiga toxin-associated haemolytic uraemic syndrome treated with eculizumab: a matched cohort study. Nephrol Dial Transplant. 2019:1-7 Paediatric matched control study providing preliminary data for efficacy of eculizumab.

66. Bauer A, Loos S, Wehrmann C, et al. Neurological involvement in children with E. coli O104:H4-induced hemolytic uremic syndrome. Paediatr Nephrol. 2014;29:1607-15.

67. Trachtman H, Austin C, Lewinski M, Stahl RAK. Renal and neurological involvement in typical Shiga toxin-associated HUS. Nat Rev Nephrol. 2012;8:658-69.

68. Nathanson S, Kwon T, Elmaleh M, Charbit M, Launay EA, Harambat $\mathrm{J}$, et al. Acute neurological involvement in diarrheaassociated hemolytic uremic syndrome. Clin J Am Soc Nephrol. 2010;5:1218-28.

69. Eriksson KJ, Boyd SG, Tasker RC. Acute neurology and neurophysiology of haemolytic-uraemic syndrome. Arch Dis Child. 2001;84:434-5.

70. Keenswijk W, Raes A, Vande Walle J. Is eculizumab efficacious in Shigatoxin-associated hemolytic uremic syndrome? A narrative review of current evidence. Eur J Pediatr. 2018;177:311-8.

71.• Johnson S (2018) Eculizumab in Shiga-toxin producing E. Coli haemolytic uraemic syndrome (ECUSTEC): a randomised, double-blind, placebo-controlled trial ECUSTEC trial PROTOCOL: VERSION 4.0, 18th An RCT that will provide high quality data on the efficacy of eculizumab used early in the treatment of STEC-HUS.

72. Garnier A, Brochard K Eculizumab in Shiga-toxin related hemolytic and uremic syndrome pediatric patients - ECULISHU. https:// clinicaltrials.gov/ct2/show/NCT02205541. Accessed 20 Aug 2019.

73. Goldstein SL. Acute kidney injury in children and its potential consequences in adulthood. Blood Purif. 2012;33:131-7.

74. Gagnadoux MF, Habib R, Gubler MC, Bacri JL, Broyer M. Longterm (15-25 years) outcome of childhood hemolytic-uremic syndrome. Clin Nephrol. 1996;46:39-41.

75. Ninchoji T, Nozu K, Nakanishi K, et al. Clinical characteristics and long-term outcome of diarrhea-associated hemolytic uremic syndrome: a single center experience. Clin Exp Nephrol. 2017;21: 889-94.

76. Small G, Watson A, Evans J, Gallagher J. Hemolytic uremic syndrome: defining the need for long-term follow-up. Clin Nephrol. 1999:352-6.

77. Cobeñas CJ, Alconcher LF, Spizzirri AP, Rahman RC. Long-term follow-up of Argentinean patients with hemolytic uremic syndrome who had not undergone dialysis. Paediatr Nephrol. 2007;22:13437.

78. Clark WF, Sontrop JM, Macnab JJ. Long term risk for hypertension, renal impairment, and cardiovascular disease after gastroenteritis from drinking water contaminated with Escherichia coli O157:H7: a prospective cohort study. BMJ. 2010;341:c6020.

79. Garg AX, Clark WF, Salvadori M, et al. Absence of renal sequelae after childhood Escherichia coli O157:H7 gastroenteritis. Kidney Int. 2006;70:807-12.

80. Perelstein EM, Grunfield BG, Simsolo RB, Gimenez MI, Gianantonio CA. Renal functional reserve compared in haemolytic uraemic syndrome and single kidney. Arch Dis Child. 1990;65: 728-31.
81. Tufro A, Arrizurieta EE, Repetto H. Renal functional reserve in children with a previous episode of haemolytic-uraemic syndrome. Pediatr Nephrol. 1991;5:184-8.

82. Kreuzer M, Sollmann L, Ruben S, Leifheit-Nestler M, Fischer D-C, Pape L, et al. Endothelial dysfunction during long-term follow-up in children with STEC hemolytic-uremic syndrome. Pediatr Nephrol. 2017;32:1005-11.

83. Trachtman H, Cnaan A, Christen E, et al. Effect of an Oral Shiga toxin-binding agent on diarrhea-associated hemolytic uremic syndrome in children: a randomized controlled trial. JAMA. 2003;290: 1337.

84. Moxley R, Francis D, Tamura M, Marx D, Santiago-Mateo K, Zhao M. Efficacy of Urtoxazumab (TMA-15 humanized monoclonal antibody specific for Shiga toxin 2) against post-diarrheal neurological Sequelae caused by Escherichia coli O157:H7 infection in the neonatal Gnotobiotic piglet model. Toxins (Basel). 2017;9:49.

85. Lopez EL, Contrini MM, Glatstein E, et al. Safety and pharmacokinetics of Urtoxazumab, a humanized monoclonal antibody, against Shiga-like toxin 2 in healthy adults and in pediatric patients infected with Shiga-like toxin-producing Escherichia coli. Antimicrob Agents Chemother. 2010;54:239-43.

86. Luz D, Chen G, Maranhão AQ, Rocha LB, Sidhu S, Piazza RMF Development and characterization of recombinant antibody fragments that recognize and neutralize in vitro Stx2 toxin from Shiga toxin-producing Escherichia coli. PLoS One. 2015. https://doi.org/ 10.1371/journal.pone.0120481.

87. Garcia-Angulo VA, Kalita A, Torres AG. Advances in the development of enterohemorrhagic Escherichia coli vaccines using murine models of infection. Vaccine. 2013;31:3229-35.

88. Marcato P, Mulvey G, Read RJ, Vander Helm K, Nation PN, Armstrong GD, et al. Immunoprophylactic potential of cloned Shiga toxin 2 B subunit. J Infect Dis. 2000;183:435-78.

89. Byun Y, Ohmura M, Fujihashi K, Yamamoto S, McGhee JR, Udaka $\mathrm{S}$, et al. Nasal immunization with E. coli verotoxin 1 (VT1)-B subunit and a nontoxic mutant of cholera toxin elicits serum neutralizing antibodies. Vaccine. 2001;19:2061-70.

90. Zhu C, Yu J, Yang Z, Davis K, Rios H, Wang B, et al. Protection against Shiga toxin-producing Escherichia coli infection by transcutaneous immunization with Shiga toxin subunit B. Clin Vaccine Immunol. 2008;15:359-66.

91. Mehr KS, Mousavi SL, Rasooli I, Amani J, Rajabi M. A DNA Vaccine against Escherichia coli O157:H7. Iran Biomed J. 2012;16:133.

92. Capozzo AVE, Pistone Creydt V, Dran G, Fernández G, Gómez S, Bentancor LV, et al. Development of DNA vaccines against hemolytic-uremic syndrome in a murine model. Infect Immun. 2003;71:3971-8.

93. Novinrooz A, Zahraei Salehi T, Firouzi R, Arabshahi S, Derakhshandeh A. In-silico design, expression, and purification of novel chimeric Escherichia coli O157:H7 OmpA fused to LTB protein in Escherichia coli. PLoS One. 2017;12:e0173761 Important potential future application for human therapy.

94. Choi KS, Kim S-H, Kim E-D, Lee S-H, Han SJ, Yoon S, et al. Protection from hemolytic uremic syndrome by Eyedrop vaccination with modified Enterohemorrhagic E. coli outer membrane vesicles. PLoS One. 2014;9:e100229.

95. Fingermann M, Avila L, Belen De Marco M, Vazquez L, Di Biase $\mathrm{DN}$, Muller AV, et al. OMV-based vaccine formulations against Shiga toxin producing Escherichia coli strains are both protective in mice and immunogenic in calves. Hum Vaccin Immunother. 2018; 14:2208-13.

Publisher's Note Springer Nature remains neutral with regard to jurisdictional claims in published maps and institutional affiliations. 